\title{
Aprovechamiento de la energía solar para mejorar las condiciones sociales de la comunidad de Loma Muleto, Bocas del Toro
}

\author{
Use of solar energy to improve the social conditions of the \\ community of Loma Muleto, Bocas del Toro
}

\author{
Tamar Jimenez ${ }^{1}$, Luis Silval, Miguel Fernández ${ }^{1}$, Vanessa Valdés ${ }^{2 *}$ \\ ${ }^{1}$ Licenciatura en Sistemas Eléctricos y Automatización-Centro Regional de Bocas del Toro- Universidad Tecnológica de \\ Panamá. ${ }^{2}$ Universidad Tecnológica de Panamá, Centro Regional de Bocas del Toro.
}

\begin{abstract}
Resumen La electrificación rural pretende mejorar las condiciones de vida y disminuir la inequidad social. El problema de la falta de energía eléctrica limita el desarrollo y obstaculiza la mejora del lugar y sus habitantes. Esto priva el desarrollo de las personas en estas áreas. Allí radica la importancia de este tema y sus soluciones que erradiquen la pobreza, la mortalidad infantil, el mejoramiento de la educación, la promoción de la equidad de género, el aumento a la atención medica de calidad y el logro de la sostenibilidad ambiental. En la provincia de Bocas del Toro, y específicamente en la comunidad de Loma Muleto la falta de energía eléctrica es evidente, ya que los lugareños son afectados en su desarrollo con respeto a la educación, la salud, la economía y tareas rutinarias del hogar. Considerando lo anterior, el objetivo es de este trabajo es evaluar el aprovechamiento de la energía solar para mejorar las condiciones sociales de la comunidad de Loma Muleto en la Provincia de Bocas del Toro. La investigación posee un enfoque cualitativo, de diseño no experimental, en donde el tipo de estudio es transversal y descriptivo. La investigación se realizó en el Distrito de Changuinola en la comunidad de Loma Muleto. Se aplicaron entrevistas a moradores de la comunidad sobre factores económicos, actividades cotidianas, educación y su sistema de salud. El análisis de datos demostró que la falta de energía eléctrica es un problema que abarca áreas importantes en la vida de los pobladores de Loma Muleto y que la necesidad de esta es urgente, ya que su ausencia no contribuye al desarrollo de la población.
\end{abstract}

Palabras clave Energía sostenible, energía renovable, potencial de aprovechamiento, zonas rurales.

\begin{abstract}
Rural electrification aims to improve living conditions and reduce social inequality. The problem of lack of electricity limits the development and hinders the improvement of the place and its inhabitants. This, in turn, deprives the development of people in these areas, therefore, the importance in the subject and its solutions that eradicate poverty, infant mortality, the improvement of education, the promotion of gender equity, the increase in quality medical care and the achievement of environmental sustainability. In the province of Bocas del Toro, and specifically in the community of Loma Muleto, the lack of electricity is evident, since the villagers are affected in their development with respect to education, health, economy and routine household tasks. Considering the above, the objective of this work is to evaluate the use of solar energy to improve the social conditions of the community of Loma Muleto in the Province of Bocas del Toro. The research has a qualitative approach, of non-experimental design, where the type of study is transversal and descriptive. The research was conducted in the District of Changuinola in the community of Loma Muleto, where interviews were given to residents of the community about economic factors, daily activities, education and their health system. The data analysis showed that the lack of electrical energy is a problem that covers important areas in the life of the residents of Loma Muleto and that the need for it is urgent, since its absence does not contribute to the development of the population.
\end{abstract}

Keywords - Sustainable energy, renewable energy, potential for use, rural areas.

*Corresponding author: vanessavvs@gmail.com

\section{Introducción}

Aproximadamente 24 millones de habitantes no tienen acceso de energía eléctrica en áreas rurales y esto priva el desarrollo de las personas en estas áreas. Por ello, es un reto afrontar este problema y erradicar la pobreza que asegure la prosperidad y el desarrollo de las comunidades rurales. 
Entre 1990 y 2010, la cantidad de personas con acceso a energía eléctrica aumentó en 1700 millones. Pero consigo la población mundial también creció y de esta manera la demanda energética accesible [1].

Uno de los problemas que ha ocasionado esto ha sido la economía global dependiente de los combustibles fósiles y el aumento de las emisiones de gases de efecto invernadero, los cuales han ocasionado cambios drásticos en nuestro sistema climático, y esto trae serias consecuencias a nivel mundial.

En el 2011 más de un $20 \%$ de la energía mundial es proporcionada por fuentes renovables, gracias a los esfuerzos de promover energía limpia. Sin embargo, esto no ha impedido que uno de cada siete personas, aún no tenga acceso a la electricidad, ya que la demanda sigue en aumento.

La razón de producir energía renovable en todo el mundo es una eficaz solución, la cual garantizaría el acceso universal de energía eléctrica para el 2030. Para lograrlo, es necesario invertir en fuentes de energía limpia, como la solar, la eólica y la térmica.

El problema de la falta de energía en zonas rurales es crítico, debido al déficit de los planeamientos de sus países gobernantes para cubrir la demanda en la regulación de la energía eléctrica. Este problema de compromiso ha hecho que la cobertura no sea la mejor para resolver la problemática de energía eléctrica.

El compromiso de empresas privadas, de los gobiernos nacionales y la cooperación internacional son claves para que se efectúen las soluciones convincentes y convenientes para los habitantes en las áreas rurales y sea un compromiso de ambas partes el progreso de esta.

La política de electrificación para zonas rurales debería ser adaptada según las necesidades de los habitantes, con tarifas que garanticen que los usuarios serán beneficiados. En los países de América Latina y el Caribe utilizan sistemas de generación aislado o micro redes y es importante mencionar, ya que se usan fuentes de generación renovables no convencionales como la energía solar, eólica y biomasa.

Un ejemplo de que el apoyo influye en gran manera es el incremento en Perú con un acceso de energía eléctrica para más de 105,000 usuarios en áreas rurales de bajos ingresos a través de un programa del Banco Mundial, el cual amplió la red eléctrica e instaló un sistema de energía solar. Este proyecto incluyó colegios, hospitales, y centros comunitarios [2].

El problema de la falta de energía limita el desarrollo y obstaculiza la mejora del lugar y sus habitantes, por ello la importancia en el tema y sus soluciones que erradiquen la pobreza, la mortalidad infantil, el mejoramiento de la educación, la promoción de la equidad de género, el aumento a la atención médica de calidad y el logro de la sostenibilidad ambiental.
El artículo 25 de la Declaración Universal de los Derechos Humanos, menciona que la energía eléctrica es vital para diferentes tareas del hogar y para el crecimiento diario de la población. Esto incluye: la alimentación, el vestido, la vivienda, la asistencia médica y los servicios sociales necesarios; el desarrollo y prosperidad de una población alimentan también la prosperidad de su país [3] figura 1.

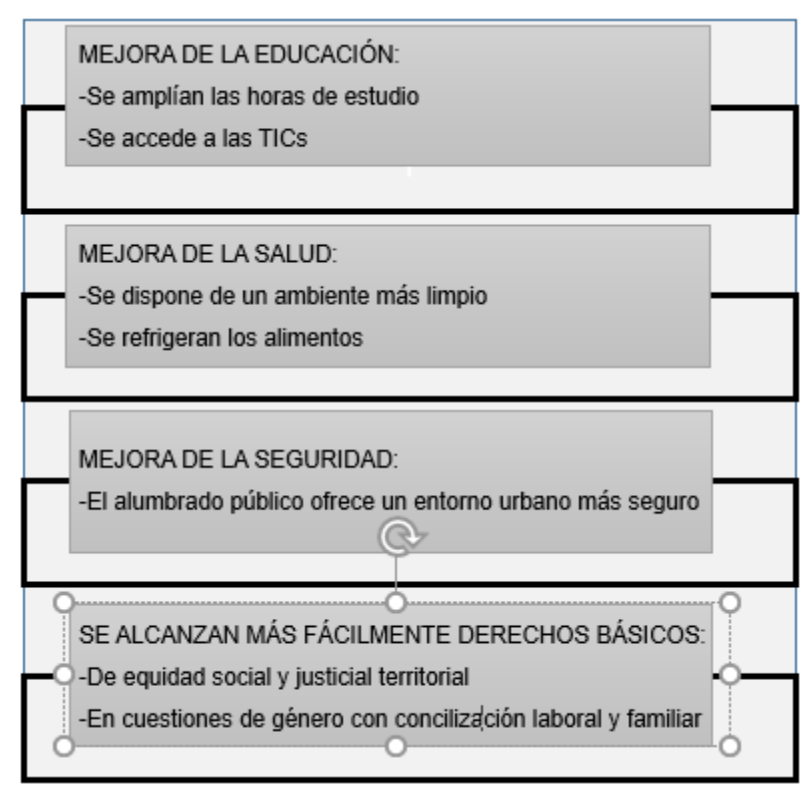

Figura 1. Aspectos valorados por la comunidad con la llegada de servicio eléctrico [4].

La Declaración Universal de los Derechos Humanos de 1948 establece también que es imposible tener prosperidad sin los servicios energéticos como, por ejemplo, el derecho al trabajo (artículo 23) en condiciones equitativas y satisfactorias, el derecho a la educación (artículo 26), al desarrollo (1986). Adicionalmente, esta Declaración indica que los estados son los principales responsables de este y quienes deben crear las condiciones favorables para lograrlo [5].

Actualmente, no existe algún tratado internacional que reconozca dicho decreto del derecho al acceso de los servicios energéticos, pero por la necesidad comienzan a surgir iniciativas que propician este interés. Sin embargo, es un hecho que muchos países están muy lejos de lograr este objetivo y que las zonas rurales aisladas son las más afectadas por esta situación.

Considerando lo anteriormente planteado, la pregunta problema de esta investigación es ¿Cómo se puede aprovechar la energía solar para mejorar las condiciones sociales de la comunidad de Loma Muleto? De esta pregunta, se deduce el siguiente objetivo: Evaluar el aprovechamiento de la energía solar para mejorar las condiciones sociales de la comunidad de Loma Muleto en la Provincia de Bocas del Toro. 


\section{Materiales y métodos}

\subsection{Tipo y diseño del estudio}

La investigación posee un enfoque cualitativo, porque no se sigue un proceso rígido y secuencial donde se pueden desarrollar hipótesis y preguntas antes, durante o después de la recolección y análisis de los datos para desarrollar la investigación, la cual tiene una gran amplitud en su proceso de comprensión pues tiene una riqueza interpretativa [6].

El diseño de investigación es no experimental, ya que, se basa en el desarrollo de un proyecto que soluciona las necesidades de energía eléctrica en áreas rurales de la provincia de Bocas del Toro, ejecutando planes de energía renovable satisfactorias para la población. Por consiguiente, se basa en la adquisición de conocimientos sobre la problemática y sus posibles soluciones [6].

El estudio es transversal, porque los datos se recogieron en un solo momento [5]. Además, es descriptiva porque busca conocer las necesidades de la población en materia de energía eléctrica en un momento dado, y estimar la magnitud y distribución de la problemática a la falta de energía eléctrica [5].

La investigación es descriptiva, ya que, es un método que implica observar y describir el desarrollo del tema donde se conocen las situaciones del problema y describe los datos para que la investigación genere un impacto en las vidas de las personas, y sus poblaciones rurales sean beneficiadas con soluciones a la falta de energía eléctrica. Según Frank Morales [6], una investigación descriptiva consiste, fundamentalmente, en caracterizar una situación concreta en la que se indican sus rasgos más peculiares o diferenciadores. Donde se conocen situaciones, costumbres y actitudes que predominan a través de la descripción exacta de las actividades y las personas.

También es prospectiva, puesto que, los datos de la información fueron recolectados en forma secuencial [6].

\section{2 Área de estudio}

La investigación se realizó en el Distrito de Changuinola Provincia de Bocas del toro, República de Panamá, figura 2.

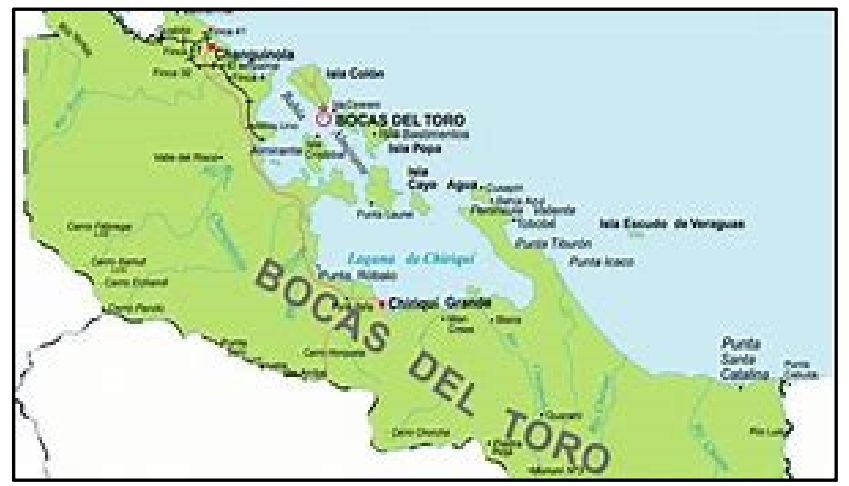

Figura 2. Provincia de Bocas del Toro [7].
La provincia de Bocas del Toro, tiene una extensión de 4 $5843,9 \mathrm{~km}^{2}$, una población de 125,461 habitantes (2010) y sus límites son: Al norte con el Mar Caribe, al sur con la provincia de Chiriquí, al este y sureste con la comarca Ngäbe-Buglé, al oeste y noroeste con la provincia de Limón de Costa Rica; y al suroeste con la provincia de Puntarenas de Costa Rica. La provincia incluye la isla Escudo de Veraguas que se encuentra en el Golfo de los Mosquitos y separada del resto por la península Valiente [8].

\subsection{Unidad de análisis y observación}

La unidad de análisis fue el Distrito de Changuinola y la unidad de observación fue la población de Loma Muleto, ubicada en la Avenida principal vía Almirante en las siguientes coordenadas: 9.3595575,-82.4909736, 5958m, a 24 minutos de camino en vehículo propio figura 3 y 4 .

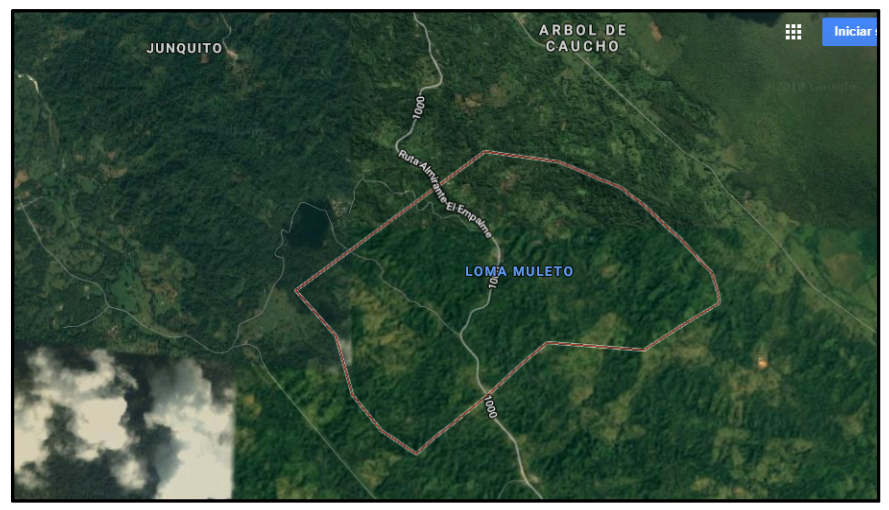

Figura 3. Localización de la comunidad de Loma Muleto [8].

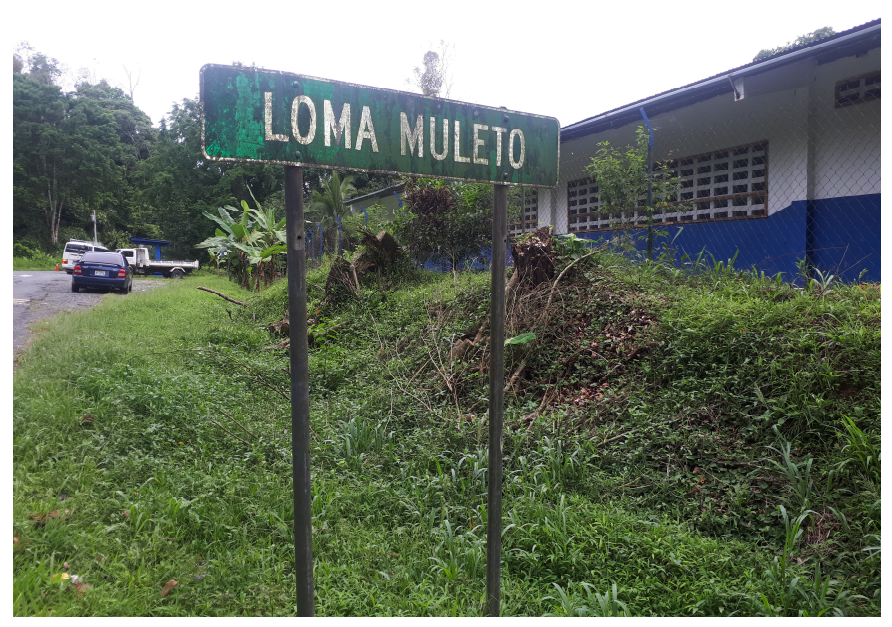

Figura 4. Entrada a la Comunidad de Loma Muleto.

\subsection{Población y muestra}

Se trabajó con una muestra intencional de diez personas de la comunidad de Loma Muleto, para dar respuesta a la variable 
de estudio. por lo tanto, no se trabajó con una muestra estadística, ya que el estudio posee un enfoque cualitativo.

\subsection{Variables}

La variable de estudio son las condiciones sociales afectadas por la falta de energía eléctrica en la comunidad de Loma Muleto. A causa de esta problemática se hizo el estudio y diseño de las instalaciones solares fotovoltaicas que permitieron la generación de electricidad para el autoconsumo de una vivienda unifamiliar, sin necesidad de depender de una red eléctrica de suministro, como alternativa exitosa.

La base sobre la cual se fundamenta los actuales sistemas fotovoltaico es el principio fotoeléctrico, mediante el cual las radiaciones de la luz solar se pueden transformar en energía eléctrica. Este efecto tiene lugar en las llamadas células fotoeléctricas, unidad básica que componen los módulos o paneles fotovoltaicos.

La radiación de luz solar se compone por partículas elementales, llamadas fotones. Estas partículas llevan asociada un valor de energía $(E)$, que depende de la longitud de onda $(\lambda)$ de la radiación.

Basándonos en la alternativa de paneles solares, es necesario la mención de los elementos que componen una instalación fotovoltaica los cuales incluyen distintos equipos, con algunos cálculos que a su vez puedan servir de guía para otras instalaciones

Estructura del sistema para la instalación fotovoltaica para una vivienda la cual pueda satisfacer las necesidades de consumo propio de electricidad figura 5.

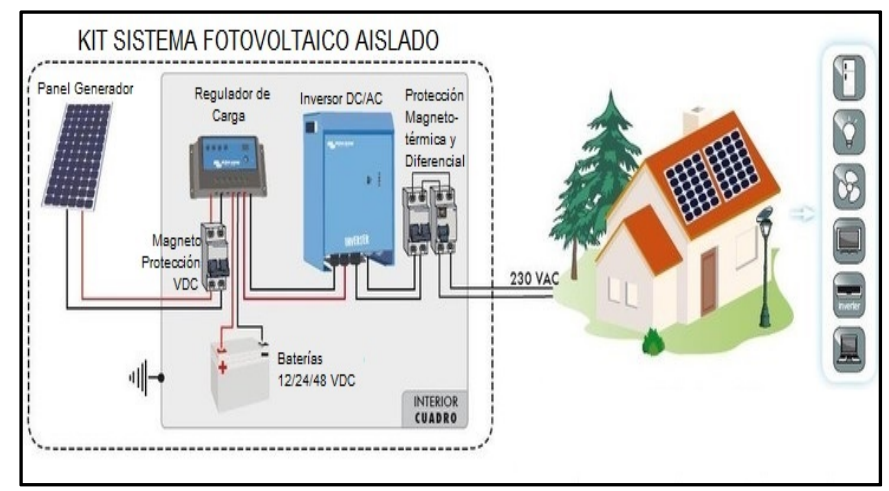

Figura 5. Sistema fotovoltaico aislado [9].

\section{Esquema de instalación}

Páneles solares: se encargan de captar la radiación solar y transformarla en electricidad, la cual genera una corriente continua, (CC), también llamada directa (DC).

El número de pánel quedará determinado por la potencia que se necesita suministrar, tomando en cuenta la forma de conexionado, ya sea en serie o paralelo.

Regulador o controlador de carga: se encarga de controlar las cargas de la batería desde los páneles, así como su descarga hacia el circuito de alimentación interior de la vivienda.
Baterías: permiten el almacenamiento de la energía que se produce durante el día con la radiación solar, también permite poder inyectar una intensidad de corriente superior a la que los propios páneles solares puedan entregar, si la instalación de la vivienda lo requiere.

Inversor o convertidor: dispositivo que permite la conversión de la corriente continua (DC) generada en los páneles fotovoltaicos en corriente alterna (AC) para que pueda ser empleada por las personas que habitan la vivienda.

\subsection{Métodos e instrumentos de recolección de datos \\ 2.6.1 Recolección de información sobre las condiciones sociales}

En el proceso de investigación se utilizó como técnica la encuesta y como instrumento la entrevista, la cual se enfocó en medir los problemas económicos, educativos y de salud causados por la escasez de energía eléctrica en áreas rurales como Loma Muleto figura 6. Este instrumento ayudó a demostrar el impacto que causa la falta de fluido eléctrico en el desarrollo de las actividades diarias de una comunidad.

En el instrumento se tomaron en cuenta factores como la educación, la salud y la economía, como indicadores indispensables para el desarrollo de una población.

Las tres preguntas estructuradas de la entrevista fueron validadas por un panel de expertos en redacción y estilo, además de docentes especialistas en electricidad, en donde, se tomaron las observaciones presentadas para mejorar las mismas.

La entrevista fue aplicada el día 10 de mayo del 2018, en la comunidad de Loma Muleto, a una población de diez personas con edades entre 14 a 80 años.

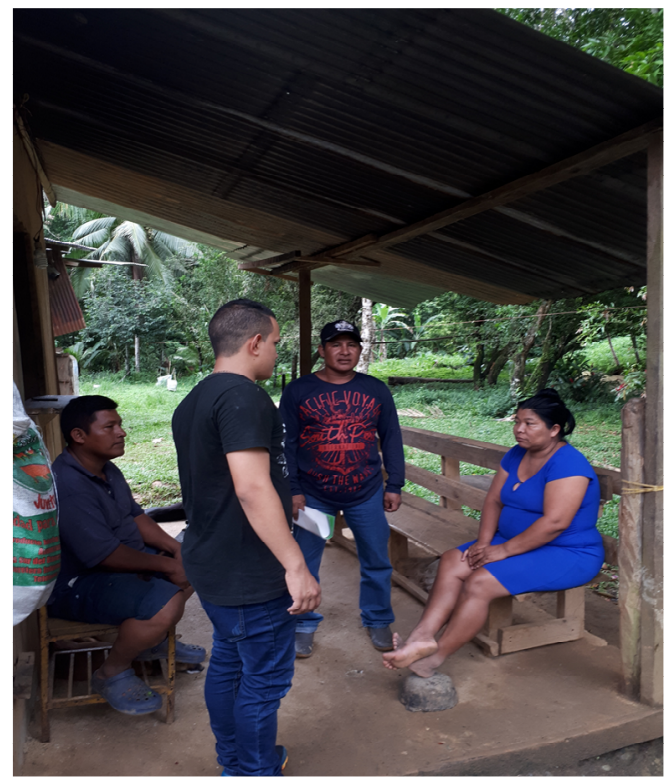

Figura 6. Entrevista a moradores de la comunidad de Loma Muleto. 


\subsubsection{Instalación del pánel solar}

Los páneles fotovoltaicos están formados por numerosas celdas que convierten la luz en electricidad. Las celdas a veces son llamadas células fotovoltaicas, Estas celdas dependen del efecto fotovoltaico por el que la energía luminosa produce cargas positiva y negativa en dos semiconductores próximos de diferente tipo, produciendo así un campo eléctrico capaz de generar una corriente.

La inversión realizada en el sistema de pánel solar fue de 1000 dólares el juego completo. Esta inversión será recuperada por el ahorro significativo de energía que representan, en unos cinco años, ya que estos páneles al ser empleados en casas pueden llegar a representar un ahorro de más de $50 \%$ con relación a lo consumido en una.

Primero para realizar la instalación del sistema se deben verificar factores como la posición del sol, carga demandada por la vivienda, voltaje de reserva permitido por el banco de baterías continuando con la instalación de las placas solares. Una vez obtenido estos datos, se conectaron las diferentes partes del sistema teniendo un regulador de corrientey batería, inverso de voltaje figura 7.

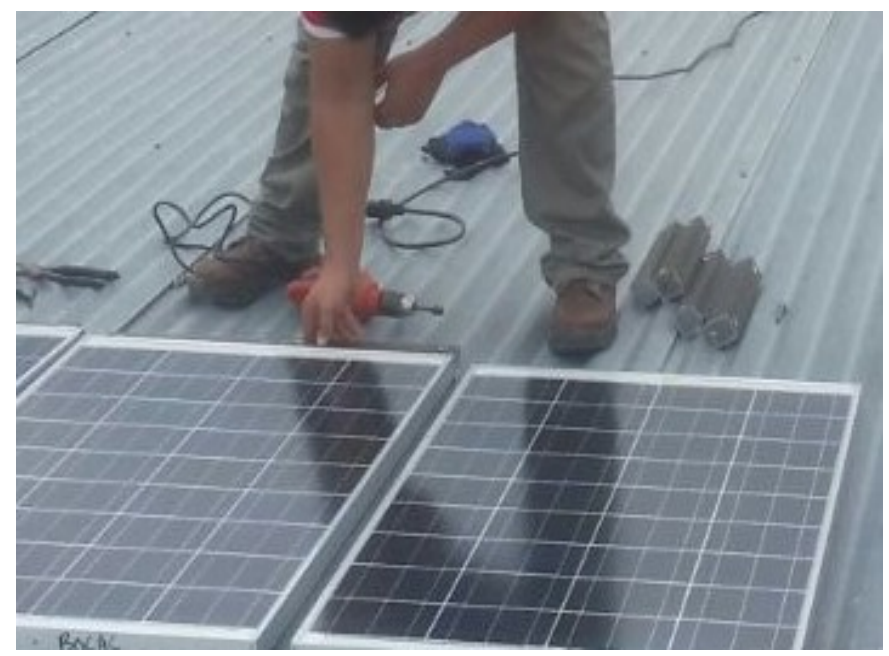

Figura 7. Instalación de placas solares.

\subsection{Análisis de datos}

Las respuestas de la entrevista fueron analizadas y agrupadas en categorías que concentran las ideas, conceptos o temas similares, siendo estos, la educación, la salud y la economía. Las mismas fueron colocadas en tablas de análisis cualitativo, para así visualizar más claramente todas las respuestas proporcionadas por los participantes de la investigación.

El panel solar utilizado fue instalado en una vivienda de Loma Muleto para evaluar las ventajas de la familia en sus condiciones sociales.

\section{Resultados y discusión}

La aplicación de la entrevista a los moradores de Loma Muleto se presenta a continuación figura 8 .

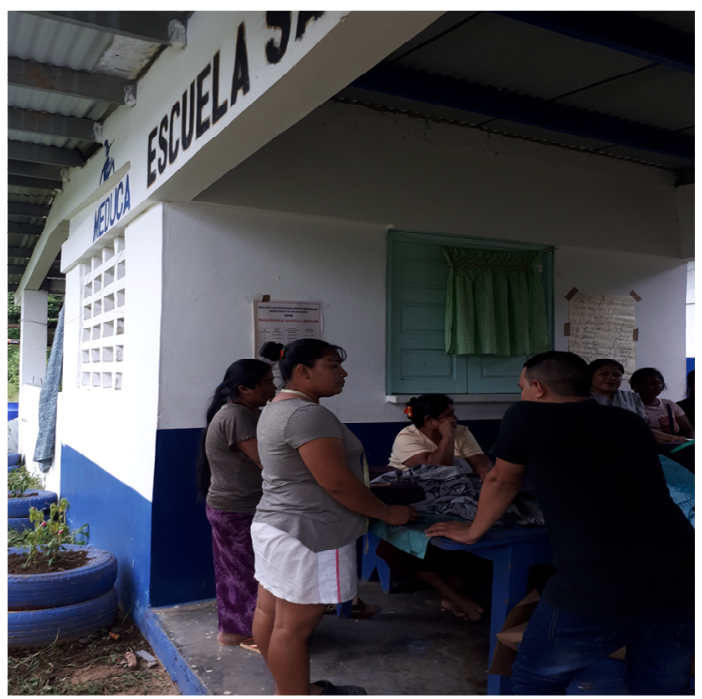

Figura 8. Entrevista aplicada a los moradores de Loma Muleto en la Escuela de San Juan, comunidad de Junquito La Gloria. Padres de familia comentan la problemática en la educación por la falta de energía eléctrica.

Los resultados son los siguientes:

\section{|Condiciones sociales}

Como se observa en la tabla 1, la falta de energía eléctrica limita el acceso a una educación adecuada, puesto que ellos explican que sin energía eléctrica no pueden estudiar durante las noches, lo que hace que disminuya su aprendizaje efectivo. De allí que, estos resultados coinciden con los estudios de [4] quienes destacan los efectos del desarrollo de la infraestructura y tecnología energética (la solar, por ejemplo), en la calidad de educación, ya que, mejoran las condiciones de estudio y la cantidad de tiempo dedicada por los estudiantes a las tareas escolares.

También, explican que como no hay energía eléctrica las clases son aburridas, porque no hay introducción de tecnología, la cual depende de la energía eléctrica.

Tabla.1. Afectación de la falta de energía eléctrica en la educación de la comunidad de Loma Muleto

Dada la pregunta ¿Cómo afecta la falta de fluido eléctrico a la educación?, los moradores respondieron lo siguiente:

- Es una gran desventaja para los estudiantes de la comunidad, ya que sus esfuerzos son disminuidos por la falta de fluido eléctrico.

- $\quad$ Sin luz es difícil abarcar todo lo necesario para el aprendizaje y profundizar en su desarrollo de manera que garantice una educación al 100\%. 
- La falta de energía eléctrica conlleva a las escasés de recursos educativos, que ayuden a tener una educación más amplia y con una gama de recursos al alcance de los estudiantes, como el uso de la internet.

- $\quad$ Se necesita del uso de dispositivos que ayuden como por ejemplo la computadora, que faciliten a los estudiantes al aprendizaje.

- Existe mucha deficiencia en el área tecnológica como proyecciones de videos y presentaciones más vistosas.

La tabla 2 muestra el grado de afectación que tiene la salud por falta de fluido eléctrico, en donde las personas de la comunidad de Loma Muleto son afectadas de manera directa, puesto que las soluciones son limitadas y no tienen el privilegio de un sistema de alta calidad y con soluciones generales para los habitantes del lugar. Estos resultados coinciden con los estudios de [4] quienes enfatizan la evolución de las condiciones de salud (recuperación de la capacidad respiratoria y problemas visuales) de las familias como resultado del empleo de electricidad y la disminución del uso de otras fuentes de energía como la leña o el carbón vegetal.

Tabla.2. Afectación de la falta de energía eléctrica en el área de la salud en la población de Loma Muleto

Dada la pregunta ¿Cómo afecta la falta de fluido eléctrico a la salud?, los moradores respondieron lo siguiente:

- La ausencia de energía eléctrica es un problema porque en altas horas de la noche cuando se necesita atención médica nos hemos visto en la necesidad de salir del lugar hasta el día siguiente, ya que la comunidad de Loma muleto se encuentra lejos del Hospital de Changuinola.

- La falta de electricidad provoca problemas para los pacientes con problemas crónicos, como es el caso de uno de los moradores de la comunidad, paciente de pulmonar crónica que necesita terapias respiratorias cuatro veces al día, y la falta de economía le impide viajar formalmente a las citas programadas.

- $\quad$ En cuanto a las medicinas, como no contamos con energía eléctrica, no podemos guardarlas para que no se dañen.

En la tabla 3, se observa que la economía de los moradores de Loma Muleto, también se encuentra afectada, puesto que, no pueden comercializar sus productos agrícolas de una forma eficiente, impidiéndole también la refrigeración de algunos productos incluso para el uso familiar. Esto refuerza las explicaciones de [4] quienes indican que la electrificación rural genera sinergias positivas entorno a la creación de actividades industriales de base agrícola en las zonas rurales figura 9.
Tabla.3. Afectación de la falta de energía eléctrica en la economía de la población de Loma Muleto

Dada la pregunta ¿Cómo afecta la falta de fluido eléctrico a la economía de la comunidad?, los moradores respondieron lo siguiente:

- La falta de energía eléctrica es una desventaja, porque no se pueden almacenar productos como las carnes, los embutidos, las bebidas, etc., que son necesarios diariamente. Esto lleva a una pérdida en los productos que con esfuerzo se pueden comprar. Esto es una pérdida para la economía de la familia.

- El no contar con energía eléctrica impide la comercialización, porque no se pueden tener cerca empresas que aseguren fuente de empleos para la mejora económica de la comunidad en general.

- La falta de energía eléctrica plantea un panorama serio de seguridad entre los residentes del área, ya que en horas nocturnas solo contamos con linternas y velas, lo cual no nos garantiza una seguridad efectiva. Además, ya se dan robos y violación a la propiedad privada.

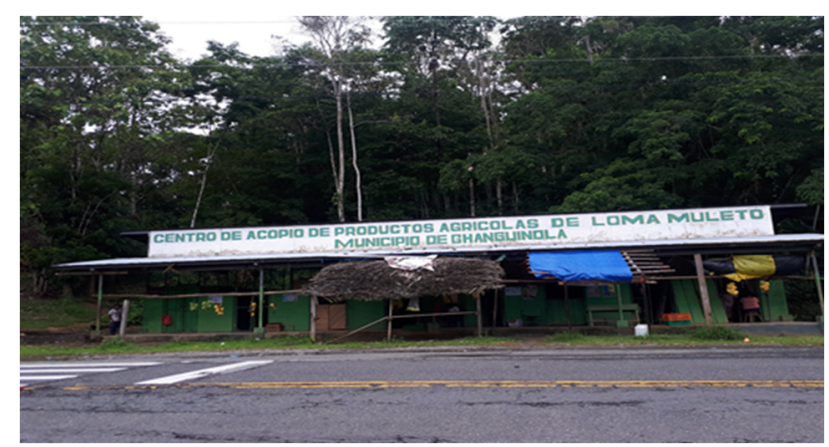

Figura 9. Centro de acopio de productos agrícolas [7].

\subsection{Pánel solar}

Al instalar un panel solar a una vivienda de la comunidad de Loma Muleto, se evidencia la satisfacción de los moradores, puesto que los beneficios de las obras de electrificación son positivos por la posibilidad de que niños y niñas puedan ampliar sus horas de estudio hasta la noche. También resaltan un mejor y mayor acceso a la información a través de las tecnologías de la información figura 10.

Además, la energía eléctrica facilita a una vivienda de la comunidad de Loma Muleto facilita el acceso a otros servicios como el agua potable y las comunicaciones. Estos servicios a una familia de la localidad provocan cambios en las formas de vida, y significan un incremento en las posibilidades $\mathrm{y}$ oportunidades de los individuos y los colectivos sociales.

Esta satisfacción coincide con los estudios de [4], quienes indican que el acercamiento del suministro eléctrico a las poblaciones alejadas de los centros urbanos no solo mejora sus condiciones de salud y educación, sino que estos servicios se apoyan directamente en la disponibilidad y accesibilidad a la electricidad. 


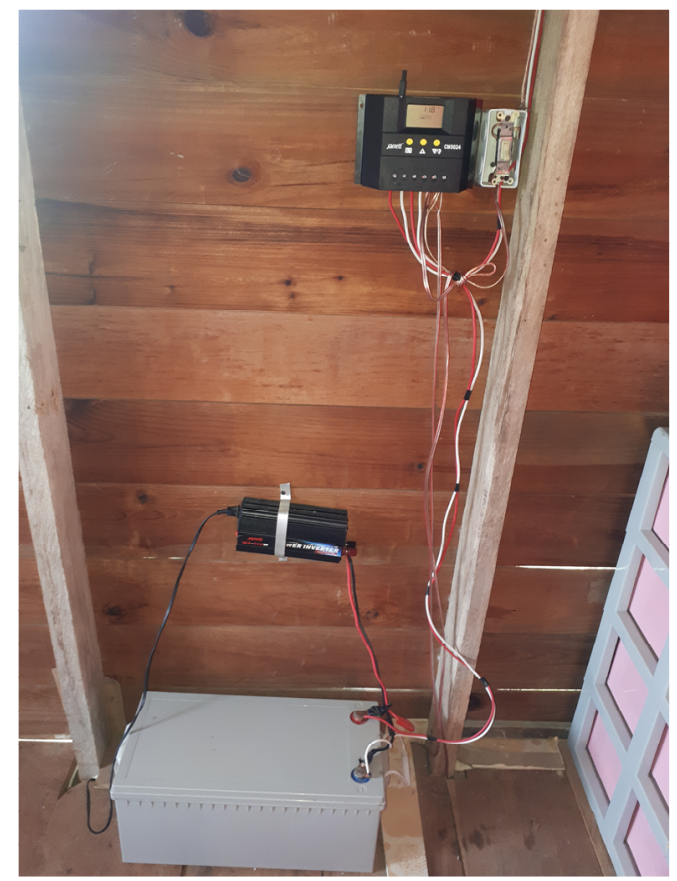

Figura 10. Sistema de generación de energía eléctrica, gracias al pánel solar.

La información obtenida referente a la falta de energía eléctrica en zonas rurales, como es el caso de la comunidad de Loma Muleto, ha demostrado que es de suma importancia resolver dicha situación. Esto es esencial, para el desarrollo en la comunidad y todos sus beneficios, los cuales incluyen la educación, salud, la economía, entre otros, ya que la ausencia de este vital medio no trae ningún avance y hace que el desarrollo sea lento y difícil para los lugareños, que se han visto con carencia en diferentes etapas de su vida y con la necesidad de buscar otros horizontes con mayores oportunidades.

Esta comunidad ha sido postergada por años; por lo que, sus habitantes carecen de los servicios ya mencionados, como la educación la cual requiere de un emprendimiento enriquecedor que lleve al buen mejoramiento y desarrollo a los estudiantes que son el futuro no solo de la comunidad sino del país en general, recibiendo clases completas en todos sus aspectos, que con beneficios eléctricos serían impartidos de manera habitual y sin ningún inconveniente tanto como para el docente como para el estudiante.

\section{Conclusiones}

Las conclusiones de esta investigación son las siguientes:

- La falta de fluido eléctrico afecta la educación de los moradores de Loma Muleto, porque sin luz es difícil abarcar y profundizar todo lo necesario para el aprendizaje. Además, limita el acceso a recursos educativos innovadores como el uso de la computadora con acceso a internet.
- La falta de fluido eléctrico afecta la salud, porque a altas horas de la noche no hay atención médica. Los pacientes deben esperar al día siguiente para ser atendidos en Changuinola donde sí hay energía eléctrica y las medicinas no se pueden almacenar, ya que no hay refrigeración.

- La falta de fluido eléctrico afecta la economía, porque no se pueden almacenar productos alimenticios que necesitan refrigeración, no hay empresas que generen ingresos familiares y no hay seguridad por los constantes robos a la propiedad privada.

- Al colocar a una casa de la comunidad un sistema de generación de energía eléctrica gracias a un panel solar, se evidenció la alegría de sus habitantes, puesto que se incrementarán sus posibilidades y oportunidades para mejorar sus condiciones sociales en cuanto a educación, salud y economía, aunque, explicaron que para que sea efectivo debe ser extendido a toda la comunidad.

Algunas de las recomendaciones son:

- Se precisa poner en marcha un sistema productivo eficiente que permita generar soluciones para estos habitantes. Se recomienda crear un programa integral que les asegure la tenencia de recursos que garanticen el desarrollo laboral y la organización comunitaria de sistemas eficaces de producción y accesos a los servicios básicos, que asegure el ingreso adecuado a todos los habitantes que lo requieren.

- Para la electrificación del lugar se ha presentado la alternativa de la instalación de páneles solares. Con este se busca generar la activación del interés y la preocupación del gobierno panameño y otras intuiciones privadas que conlleven a actividades que financien la cobertura de energía eléctrica sostenible, de manera que la comunidad avance en su desarrollo de manera que sea un ejemplo para la expansión a otros lugares donde no existe el servicio de energía eléctrica.

\section{AGRADECIMIENTO}

A Dios por permitirnos culminar con éxito esta investigación. A la Doctora Vanesa Valdés, quien estuvo presente en todo momento durante la realización de este trabajo, y a quien nos gustaría expresar nuestro más profundo agradecimiento, por hacer posible la realización de este estudio. Además, le agradecemos su paciencia, tiempo y dedicación para que esto saliera de manera exitosa.

A la Jornada de Iniciación Científica por hacernos creer en nosotros para hacer una investigación, y a la población de Loma Muleto por su colaboración, y a nuestras familias por su apoyo. 
Jimenez (et al): Aprovechamiento de la energía solar para mejorar las condiciones sociales de la comunidad de Loma Muleto, Bocas del Toro

\section{REFERENCIAS}

[1] R. Kaur, "energia asequíble y sostenible," programas de las naciones unidas para el desarrollo.

[2] B. Mundial, "Electricidad para las comunidades rurales en el Perú," Banco Mundial, 24 septiembre 2014.

[3] N. Unidas, "declaracion universal de los derechos humanos," Naciones Unidas.

[4] D. Mendieta and J. Escribano, "Electricidad, desarrollo rural y buen vivir," México, 2015.

[5] L. I. y. J. Eisman, "La electrificación sostenible de zonas rurales aisladas de países en desarrollo mediante microsistemas eléctricos renovables," cuadernos de energía, 2009.
[6] R. Hernández Sampieri, C. Fernández Collado and P. Baptista Lucio, Metodología de la investigación, Sexta ed., España: McGraw-Hill / Interamericana de España, S.A, 2014, p. 613.

[7] Galería de Provincias - Panamá.

[8] google maps.

[9] H. R. Galbarro, Instalación solar fotovoltaica para Vivienda, 2018.

[10] Wikipedia, "Wikipedia," WikiMedia project, 3042018. [Online]. Available: https://es.wikipedia.org/wiki/Provincia_de_Bocas_del_Toro. [Accessed 13 Mayo 2018]. 